\title{
Macrophage conditioned media promotes adipocyte cancer-association, which in turn stimulates breast cancer proliferation and migration
}

\section{Karin A. Vallega}

Florida State University

Dale B. Bosco

Florida State University

Yi Ren

Florida State University

Qing-Xiang Sang ( $\nabla$ qsang@fsu.edu )

Florida State University https://orcid.org/0000-0001-8828-0569

Research article

Keywords: Breast cancer, macrophages, adipocyte, cancer-association, inflammation, obesity

Posted Date: December 18th, 2020

DOl: https://doi.org/10.21203/rs.3.rs-130272/v1

License: (c) (i) This work is licensed under a Creative Commons Attribution 4.0 International License. Read Full License 


\section{Abstract \\ Background}

Breast cancer is the most common cancer in women and the leading cause of female cancer deaths worldwide. Obesity causes chronic inflammation and is a risk factor for post-menopausal breast cancer and poor prognosis. Obesity is known to trigger increased infiltration of macrophages into adipose tissue, yet little research has focused on the effects of macrophages in the early stages of breast tumor development in obese patients. In this study, the effects of pro-inflammatory macrophages on breast cancer-adipocyte crosstalk were investigated.

\section{Methods}

An innovative human cell co-culture system was used to model the paracrine interactions among adipocytes, macrophages, and breast cancer cells, and how they can facilitate tumor progression. The effects on human breast cancer cells were examined using cell counts and migration assays. Quantitative reverse-transcription polymerase chain reaction (qRT-PCR) was used to measure the expression levels of several cytokines and proteases to analyze adipocyte cancer-association.

\section{Results}

Macrophage conditioned media intensified the effects of breast cancer-adipocyte crosstalk. More specifically, adipocytes became delipidated and increased production of pro-inflammatory cytokines, even in the absence of breast cancer cells, although the expression levels were highest with all three cell components. As a result, co-cultured breast cancer cells became more aggressive, with increased proliferation and migration potential when compared to adipocyte-breast cancer cell co-cultures treated with unconditioned media.

\section{Conclusions}

Macrophage conditioned media promotes adipocyte cancer-association and production of proinflammatory factors. These macrophage-adipocyte paracrine interactions promote human breast cancer cell proliferation and migration. Thus, macrophages may contribute to adipocyte inflammation and cancer-association and promote breast cancer progression.

\section{Introduction:}

Obesity is a growing problem affecting an estimated 650 million adults worldwide ${ }^{1}$. In the United States, approximately forty percent of adults were classified as obese in $2016^{2}$. Obesity increases the risk of 
several types of cancer, including post-menopausal breast cancer ${ }^{3}$. It is also a poor prognosis marker for several cancers and causes increased death rates ${ }^{4-6}$. It is estimated that $14 \%$ of cancer death in men and $20 \%$ in women are related to obesity ${ }^{5}$.

In breast cancer, tumor cells are in close proximity to adipocytes. Adipocytes in contact with tumor cells show distinctive phenotypic changes and altered secretion profiles, which have been termed cancerassociated adipocytes (CAAs) ${ }^{7}$. CAAs undergo a reduction in size and lipid content, and upregulate

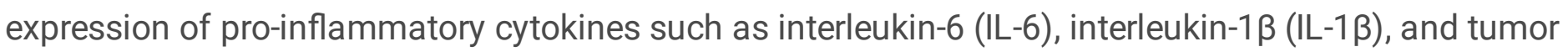
necrosis factor- $a(T N F-a)^{7}$. Proteases that can remodel the extracellular matrix (ECM), such as matrix metalloproteinase-11 (MMP-11), are also upregulated ${ }^{7}$. CAAs, in turn support tumor growth, migration, and invasion $7,8,9$, resulting in a process known as breast cancer - adipocyte crosstalk.

Obesity also results in increased infiltration of macrophages into adipose tissue ${ }^{10,11,12}$. Macrophages recruited into adipose tissue are pro-inflammatory and contribute to the chronic inflammation seen in obese people ${ }^{12}$. Many studies have demonstrated that established tumors can cause macrophages to undergo anti-inflammatory supportive polarization ${ }^{13,14}$. These anti-inflammatory macrophages then benefit tumor growth and survival by shielding cancer from the immune system ${ }^{13,14}$. However, little research has focused on the effects of macrophages in the early stages of tumor development in obese patients. In this study, we investigated the effects of pro-inflammatory macrophages on breast canceradipocyte crosstalk.

\section{Materials And Methods:}

\section{Cell Culture:}

Adult human mesenchymal stem cells (hMSCs) were obtained from the Tulane Center for Gene Therapy. Human MSCs were grown in Minimum Essential Medium, Eagle Alpha Modification (a-MEM; Sigma, St. Loius, MO; M0644) supplemented with $20 \%$ fetal bovine serum (FBS; Seradigm, Radnor, PA) and $2.2 \mathrm{~g} / \mathrm{L}$ sodium bicarbonate. An L-glutamine, penicillin, and streptomycin cocktail (Sigma; G1146) was also added for a final concentration of $200 \mathrm{mM} \mathrm{L}$-glutamine, 10,000 units $/ \mathrm{mL}$ penicillin, and $100 \mathrm{mg} / \mathrm{mL}$ streptomycin. The human U937 cell line was obtained from American Type Culture Collection (ATCC, Manassas, VA; CRL-1593.2) and grown in RPMI-1640 media (Sigma; R8755) supplemented with 10\% FBS, $2.0 \mathrm{~g} / \mathrm{L}$ sodium bicarbonate, and L-glutamine, penicillin, and streptomycin cocktail. MCF-7 human breast cancer cells were obtained from ATCC (HTB-22) and grown in a-MEM supplemented with 10\% FBS, $2.2 \mathrm{~g} / \mathrm{L}$ sodium bicarbonate, and L-glutamine, penicillin, and streptomycin cocktail. MDA-MB-231 human breast cancer cells were obtained from ATCC (HTB-26) and grown in a-MEM supplemented with 10\% FBS, $2.2 \mathrm{~g} / \mathrm{L}$ sodium bicarbonate, and L-glutamine, penicillin, and streptomycin cocktail. Cells were maintained in a fully humidified incubator at $37 \otimes \mathrm{C}$ with $5 \% \mathrm{CO}_{2}$.

\section{Differentiation of hMSCs into Adipocytes:}


Human MSCs were plated at 1,000 cells/ $\mathrm{cm}^{2}$ in 6-well plates for 2D cell culture and 2,000 cells per well in 96-well plates for 3D spheroid culture. Monolayer cultures were maintained in the a-MEM media described above until $70 \%$ confluent. Then, adipocyte differentiation was induced through the addition of $0.5 \mu \mathrm{M}$ dexamethasone (Sigma; D4902), $0.5 \mu \mathrm{M}$ isobutylmethylxanthine (Sigma; I7018), and $50 \mu \mathrm{M}$ indomethacin (Sigma; 17378) to the culture media ${ }^{15,16}$. After 21 days of differentiation, cells were used for further experimentation.

\section{Differentiation of U937 Cells into Macrophages:}

U937 cells were induced towards a macrophage phenotype via the addition of phorbol myristate acetate (PMA; BioVision, San Francisco, CA; 1544-5) to the media ${ }^{17,18}$. Various concentrations $(0-100 \mathrm{ng} / \mathrm{mL})$ and timepoints (24 or $48 \mathrm{hrs}$ ) were examined to determine optimal differentiation (Fig. S1). Following treatment, cells were cultured for an additional $72 \mathrm{hrs}$ in untreated media. Differentiation was determined by assessing the morphology and attachment of the cells. Non-adherent cells were removed, and adherent cells were counted using a Cedex HiRes cell analysis system (Innovatis Inc., Fairfax, VA). $100 \mathrm{ng} / \mathrm{mL}$ PMA for 48 hours was determined to yield the most differentiated cells, and subsequently used for all experiments.

\section{Macrophage Conditioned Media (CM) Preparation:}

Once differentiation conditions were optimized, 1 million U937 cells $/ \mathrm{mL}$ were plated in $10 \mathrm{~cm}$ dishes. $100 \mathrm{ng} / \mathrm{mL}$ PMA was added. After 48 hours, PMA was removed and cells were cultured in untreated media for 72 hours. Then, media was changed and conditioned for 24 hrs. Media was then collected and filtered through a 0.2 micron filter. Conditioned media (CM) was stored at -20खC until used.

\section{Breast Cancer-Adipocyte Co-Culture:}

MCF-7 breast cancer cells were co-cultured with adipocytes in macrophage conditioned media (Fig. 1). 24 well dishes were used for breast cancer cell proliferation and migration assays. 6 well dishes were used for RT-PCR experiments. The corresponding size transwells were used to keep the cell types apart. MCF-7 cells were plated at $2 \times 10^{4}$ cells $/ \mathrm{cm}^{2}$. Adipocytes were plated at $2 \times 10^{4}$ cells in 24 wells, which corresponded to 10 spheres of 2,000 cells/sphere. For qRT-PCR, adipocytes were plated in normal 2D culture as described above. Macrophage conditioned media was used 1:1 with regular growth media. MCF-7 cells co-cultured with adipocytes in unconditioned media served as controls. MCF-7 cells were also grown alone in macrophage conditioned media as an additional control. After 3 days, the system was taken apart and each cell type was examined individually. All co-cultures were left together for 3 days unless specified otherwise. The same methods were used for co-culture experiments using the MDA-MB231 cell line.

\section{Breast Cancer Cell Counts:}

All cell counts were made using a Cedex HiRes cell analysis system. Trypan blue was used for live/dead staining. All experiments were run in triplicate. To determine viability, live cell counts were divided by total 
cell counts for each condition.

\section{Wound Healing Assay:}

After 3 days in co-culture, the adipocytes were removed and a scratch in the shape of a cross was made on the confluent breast cancer cell layer using a $200 \mu \mathrm{L}$ pipette tip. The cells were washed with PBS and fresh media was added. Pictures were taken, centered on the center of the cross for reference, and the cells were left to recover for 24 hours, after which pictures were taken again. The area was quantified using the MRI wound healing tool in ImageJ and represented as percent of wound closed after 24 hours. All experiments were run in triplicates.

\section{Real Time Quantitative reverse-transcription polymerase chain reaction (qRT-PCR):}

After PMA treatment, macrophage cells were left to recover for 48 hours and then harvested for qRT-PCR. RNA was isolated from the cells using the E.Z.N.A. Total RNA Kit I (Omega Bio-Tek, Norcross, GA; R6834) and purified with the RNA Clean \& Concentrator Kit (Zymo Research, Irvine, CA; R1013). 1 ug of RNA was converted to cDNA using the qScript cDNA SuperMix (Quanta Bioscience, Beverly, MA; 95048 ). $100 \mathrm{ng} / \mu \mathrm{L}$ cDNA was used as template for quantitative PCR on a 7500 Fast Real Time PCR system (Applied Biosystems, Foster City, CA) using a SYBR green PCR master mix (Applied Biosystems; 4344463). The PCR was run as follows: one cycle of 2 minutes at $50{ }^{\circ} \mathrm{C}$ and 10 minutes at $95^{\circ} \mathrm{C}$, then 40 cycles of 15 seconds at $95^{\circ} \mathrm{C}, 30$ seconds at $55^{\circ} \mathrm{C}$ and 30 seconds at $68^{\circ} \mathrm{C}$. Relative gene expression was determined using the $2^{(-\Delta \Delta C t)}$ method $^{19}$. Primers were ordered from Eurofins (Luxembourg) and GAPDH was used as an endogenous control.

For adipocytes, 2D cultures were placed either in co-culture with MCF-7 cells or alone, and either in macrophage conditioned media or non-conditioned media. The adipocytes were harvested after 6 and 24 hours and the samples were prepped for qRT-PCR by the same method described above. 
Table 1

Primers used for qRT-PCR.

\begin{tabular}{|lll|}
\hline Gene & Forward Primer & Reverse Primer \\
\hline CD 11b & 5'-CTTCCAGGTTCTGGCTCCTTC-3' & 5'-TCTCTTGGAAGGTCATTGCGTT-3' \\
\hline MCP-1 & 5'-TCTCAAACTGAAGCTCGCACT-3' & 5'-GGGAATGAAGGTGGCTGCTA-3' \\
\hline IL-6 & 5'-CCTGCAGAAAAAGGCAAAGAATC-3' & 5'-GAGTTGTCATGTCCTGCAGCC-3' \\
\hline RETN & 5'-TGCAGGATGAAAGCTCTCTGTCTC-3' & 5'-CCTGGATCCTCTCATTGATGGC-3' \\
\hline TNF-a & 5'-TGGGATCATTGCCCTGTGAG-3' & 5'-GGTGTCTGAAGGAGGGGGTA-3' \\
\hline IL-1ß & 5'-CAGGCTGCTCTGGGATTCTC-3' & 5'-AAGTCATCCTCATTGCCACTGT-3' \\
\hline AdipoQ & 5'-ATGGCCCCTGCACTACTCTA-3' & 5'-CAGGGATGAGTTCGGCACTT-3' \\
\hline LEP & 5'-CCCTGGAGTGCAGTTTCCAA-3' & 5'-TGCTCAGATGAACCCAACCC-3' \\
\hline PAI-1 & 5'-TTGCAGGATGGAACTACGGG-3' & 5'-GTGGCAGGCAGTACAAGAGTGA-3' \\
\hline MMP-3 & 5'-CCATCTCTTCCTTCAGGCGT-3' & 5'-ATGCCTCTTGGGTATCCAGC-3' \\
\hline MMP-11 & 5'-ATGAATTTGGCCACGTGCTG-3' & 5'-CGAAAGGTGTAGAAGGCGGA-3' \\
\hline
\end{tabular}

\section{Statistical Analysis:}

Experiments were performed with at least three independent samples per data point. Data analysis was done in Excel using Student's T-test. Proliferation, viability, migration, differentiation and qRT-PCR results are expressed as the mean \pm standard error.

\section{Results:}

\section{Quantitative RT-PCR Analysis of U937 Monocyte-derived Macrophages:}

Differentiated U937 cells were analyzed for expression of macrophage marker CD11 $b^{17}$, which was greatly increased after PMA treatment $(p$-value $=0.0004)($ Fig. $2 A)$. Differentiated cells also showed changes in morphology (Fig. 2B) consistent with a macrophage phenotype. More specifically, after differentiation U937 cells became adherent and displayed pseudopodia.

Expression levels of several cytokines of interest were quantified using qRT-PCR (Fig. 2C). Differentiated macrophages showed significantly increased levels of TNF-a $(p-v a l u e=0.0006)$ and IL-1 $(p$-value $=$ $0.004)$, and significantly decreased expression levels of IL-6 ( $p$-value $=0.025)$ and RETN ( $p$-value $=$ 0.0007) (Fig. 2C). However, though IL-6 and RETN were decreased, these pro-inflammatory cytokines were still produced by the macrophages. This expression profile suggests the macrophages are a classical, M1 pro-inflammatory phenotype. 


\section{Breast cancer cell proliferation and migration:}

Using the co-culture model described in Fig. 1, the effects of macrophage $\mathrm{CM}$ on breast cancer cells were examined. MCF-7 cells in co-culture with adipocytes in macrophage CM showed a significant increase in cell number after 3 days, when compared to both cells grown in CM alone (0.001) and in co-culture with nonconditioned media ( $p$-value $=0.004)(F i g .3 A)$. Cell viability was similar between all conditions (Fig. S2). MCF-7 cells co-cultured with adipocytes in macrophage $\mathrm{CM}$ also showed increased migration capabilities when compared to cells co-cultured in nonCM ( $p$-value $=0.02)$ (Fig. 3B).

The effects of adipocyte co-culture and macrophage $\mathrm{CM}$ were then investigated with the more aggressive, triple-negative breast cancer cell line, MDA-MB-231 for comparison (Fig. S3). Unlike the MCF-7 cells, MDAMB-231 cells did not show a significant increase in cell proliferation ( $p$-value $=0.098)$ or migration $(p-$ value $=0.4$ ) when macrophage $\mathrm{CM}$ was added to the co-culture system (Fig. S3). Viability was again similar across all conditions (Fig. S4). However, MDA-MB-231 cells did show increased proliferation when in co-culture with adipocytes compared to alone in macrophage CM ( $p$-value $=0.03$ ) (Fig. S3).

\section{Quantitative RT-PCR Analysis of Adipocytes}

Quantitative RT-PCR analysis was used to measure the mRNA expression levels of several cytokines and proteases of interest that indicate delipidation of adipocytes (Fig. 4). Adiponectin (AdipoQ), which is inversely correlated with fat mass, was significantly increased after 24 hours in co-culture with MCF-7 BRCA cells with macrophage CM compared to co-culture in nonCM ( $p$-value $=0.02)(F i g .4 A)$. This indicates a decrease in the lipid content of the adipocytes when macrophage CM is added to the coculture. Plasminogen activator inhibitor-1 (PAl-1), which is directly correlated with fat mass, decreased significantly after 24 hours $(p$-value $=0.018)$. All the measured pro-inflammatory cytokines $(I L-1 \beta, I L-6$, and TNF- $a$ ) increased when macrophage CM was added to the co-culture system. IL- 6 ( $p$-value $=0.014)$ and TNF- $a(p$-value $=0.0005)$ gene expression spiked early, after 6 hours, while IL-1 $\beta(p$-value $=0.0003)$ continued to increase over 24 hours. TNF-a can cause delipidation of adipocytes ${ }^{20}$, and levels increased early, before the rise in AdipoQ, which suggests decreased lipid content in the co-culture exposed to macrophage CM. qRT-PCR analysis was also used to test for MMP-3 and MMP-11 levels (Fig. 4B). MMP-3 and MMP-11 are both negative regulators of adipogenesis, and MMP-11 can cause delipidation of adipocytes ${ }^{21,22}$. MMP-3 expression levels increased significantly after both $6(p$-value $=0.007)$ and 24 hours $(p$-value $=0.002)$. MMP-11 also increased after 24 hours ( $p$-value $=0.007)$.

Additionally, adipocytes cultured alone with or without macrophage CM were analyzed by qRT-PCR to study the direct effects of macrophage CM on adipocytes (Fig. 5). AdipoQ and leptin (LEP) mRNA expression levels were similar across both conditions (Fig. 5A). However, expression of pro-inflammatory cytokines, IL-6, TNF- $a$, and IL-1 $\beta$, increased in response to macrophage CM. IL-1 $\beta$ showed the largest difference after 24 hours $(p-v a l u e=0.00001)$, while IL- $6(p$-value $=0.0007)$ and TNF- $a(p-v a l u e=0.0004)$ peaked at 6 hours. PAl-1 expression decreased significantly after 24 hours ( $p$-value $=0.006)$. Monocyte chemoattractant protein-1 (MCP-1) expression was not altered. 
Macrophage $\mathrm{CM}$ also increased expression of MMPs in adipocytes cultured without breast cancer cells (Fig. 5B). MMP-3 was significantly higher after 6 hours ( $p$-value $=0.00001)$ and continued to increase over 24 hours $(p$-value $=0.0000004)$. MMP-11 expression was significantly increased after 24 hours $(p$ value $=0.003)$.

\section{Discussion:}

Obesity causes systemic low-grade inflammation, which in turn increases cancer incidence and mortality. In breast tissue, obesity also results in increased infiltration of macrophages that contribute to inflammation. Many studies have shown that cancer cells can cause delipidation and cancer-association of adipocytes in the breast cancer microenvironment ${ }^{7,23}$. The ability of established tumors to stimulate $\mathrm{M} 2$ conversion in macrophages is also well documented ${ }^{24}$. However, the effects of nascent proinflammatory M1 macrophages on breast cancer-adipocyte crosstalk in early tumorigenesis is largely unknown.

Macrophage conditioned media has varying effectiveness upon different types of breast cancer cells.

To more accurately model the human breast cancer microenvironment, we developed a novel co-culture system that utilized only human derived cells and could model the breast cancer-adipocyte crosstalk in an obese and lean patient. Our model could also examine the contribution of infiltrating macrophages. The luminal A cell line, MCF-7, was principally used since it is hormone receptor positive and not metastatic. This non-aggressive cell line is a better representation of early tumorigenesis, which is usually less aggressive than an established tumor. MCF-7 cells grown in co-culture with macrophage CM showed higher proliferation and migration compared to breast cancer cells in co-culture without macrophage CM (Fig. 3). MCF-7 cells were also cultured alone in macrophage CM, which did not cause increased cell proliferation (Fig. 3A). Therefore, this effect is not due solely to the pro-inflammatory cytokines secreted by the macrophages, such as IL-1 $\beta$ and TNF- $\alpha$ (Fig. 2C), which can benefit tumor cell proliferation. Instead, the macrophage secreted factors affect the adipocytes, which in turn affect the breast cancer cells. MCF-7 cells grown in co-culture without macrophage $\mathrm{CM}$ also showed higher proliferation than the cancer cells alone, which agrees with previous studies that show increased tumor growth as a result of breast cancer-adipocyte crosstalk ${ }^{7,9}$. However, our study shows this effect is even stronger when macrophage $\mathrm{CM}$ media is added. This indicates macrophages that infiltrate into breast adipose tissue could aid in early tumorigenesis, before the tumor cells stimulate an M1 to M2 conversion.

This study's main focus is on the effect of M1 macrophage infiltration in priming an inflammatory microenvironment in early tumorigenesis. However, to see if this would also affect a more aggressive, established tumor, the breast cancer proliferation and migration studies were repeated using the MDAMB-231 cell line (Fig. S3). MDA-MB-231 is a triple-negative, highly aggressive and metastatic breast cancer cell line. In co-culture, adipocytes again promoted the growth of the cancer cells. However, in contrast to MCF-7 cells, macrophage CM did not appreciably increase MDA-MB-231 cell growth or migration. One possible explanation for the observed difference is the estrogen receptor status of these 
cell lines. MCF-7 cells are estrogen receptor positive, while MDA-MB-231 cells are estrogen receptor negative. Adipocytes synthesize estrogen through aromatase mediated metabolism of androgen precursors in post-menopausal women ${ }^{30}$. Macrophage derived proinflammatory mediators can also induce aromatase and estrogen dependent gene expression in adipocytes ${ }^{31}$. Therefore, estrogen overexpression by adipocytes could be one mechanism by which cancer-associated adipocytes contribute to breast cancer progression.

Macrophage conditioned media increases the pro-inflammatory character of adipocytes.

The expression levels of several cytokines associated with inflammation and breast cancer were highly up-regulated by astrocytes, both in co-culture and alone, in response to macrophage CM. For instance, there was marked increases in expression of the pro-inflammatory cytokines, IL-1 $\beta$, TNF- $a$, and IL- 6 . This is consistent with another study by Permana et al., which also showed that murine RAW 264.7 macrophage conditioned media could induce 3T3-L1 adipocyte inflammation ${ }^{29}$. The co-culture system used in our study consisted of only human cell lines, establishing that these effects are present in human cell interactions, and can have important implications in human breast cancer progression and prognosis.

Curiously however, increased expression of adiponectin, an anti-inflammatory cytokine that is secreted solely by mature adipocytes and inversely correlated to fat mass, seemed to be more dependent upon coculture with breast cancer cells than addition of macrophage CM. Increased adiponectin is an indicator of delipidation, which is one of the hallmarks of adipocyte cancer-association ${ }^{7,20}$. TNF-a has also been reported to cause delipidation of adipocytes and may promote the expression of adiponectin ${ }^{23,24}$. Yet when adipocytes were cultured alone in macrophage $\mathrm{CM}$, there was no significant increase in adiponectin, unlike in co-culture, even though TNF-a was increased. Consequently, it would seem additional breast cancer cell related factors play a substantial role in adiponectin expression, and all three cell types work in concert to cause adipocyte cancer-association and promote tumor aggressiveness.

Macrophage conditioned media increases matrix metalloproteinase expression in adipocytes.

Macrophage CM also increased MMP-3 and MMP-11 expression by adipocytes in adipocyte-breast cancer co-culture (Fig. 4B). MMP-11 is a negative prognosis marker in several human cancers ${ }^{28}$ and can also cause delipidation of adipocytes ${ }^{20}$. Andarawewa et al. found that MMP-11 was expressed in human adipocytes proximally located to invasive cancer cells, but not distally located adipocytes ${ }^{20}$. In addition, this same study found these adipocytes at the tumor invasive front that expressed MMP-11 were reduced in size. In studies examining the role of MMPs in normal adipogenesis, MMP-11 and MMP-3 deficient mice developed more adipose tissue, indicating a negative regulatory role 21,22 . The increased expression on MMP-11 and MMP-3 further indicate that macrophage CM can increase adipocyte delipidation and cancer-association. Finally, while MMP-11 expression by adipocytes, both in co-culture and alone, was similarly affected by macrophage CM, there appeared to be an additive affect on MMP-3 expression. 
Although MMP-3 and MMP-11 are structurally and functionally similar, both being stromelysins and negative regulators of adipogenesis, their expression may require different signaling mechanisms.

\section{Conclusion:}

In conclusion, our results suggest that macrophage derived factors can exacerbate the effects of human breast cancer cell-adipocyte crosstalk. Macrophage CM resulted in increased breast cancer cell proliferation and migration, and adipocyte cancer-association. The influx of macrophages into adipose tissue in the obese patients contributes to the inflammation caused by adipocytes, and may prime the microenvironment for tumorigenesis. When breast cancer cells are added into this system, adipocytes produce even more pro-inflammatory cytokines and show signs of delipidation, and promote tumor cell aggressiveness. These results are of particular importance for obese breast cancer patients, who have increased macrophage infiltration into adipose tissue. The effects of macrophages on this crosstalk may help account for the worse prognosis in obese breast cancer patients. Additionally, these findings represent potential new treatment or drug targets for obese breast cancer patients. Limiting macrophage infiltration, or reducing the secretion of macrophage proinflammatory cytokines, could help reduce inflammation in breast tissue and make the microenvironment less favorable for tumorigenesis.

\section{Abbreviations}

a-MEM

Minimum Essential Medium, Eagle Alpha Modification

AdipoQ

Adiponectin

BRCA

Breast cancer

CAA

Cancer-associated adipocytes

CD11b

Cluster of differentiation molecule 11B

$\mathrm{CM}$

Conditioned media

ECM

Extracellular matrix

FBS

Fetal bovine serum

GAPDH

Glyceraldehyde 3-phosphate dehydrogenase

hMSCs

Human mesenchymal stem cells 
IL-1 $\beta$

Interleukin-1 $\beta$

IL-6

Interleukin-6

LEP

Leptin

MMP-3

Matrix metalloproteinase-3

MMP-11

Matrix metalloproteinase-11

nonCM

Non-conditioned media

PAl-1

Plasminogen activator inhibitor-1

PMA

Phorbol myristate acetate

RETN

Resistin

$\mathrm{RQ}$

Relative quantification

TNF-a

Tumor necrosis factor-a

\section{Declarations}

Ethics approval and consent to participate: Not applicable. This work used commercially and publicly available de-identified human cell lines established many years ago.

Consent for publication: Not applicable

Availability of data and materials: The datasets generated and analyzed during the current study are available in this publication and supplemental files.

Competing interests: The authors declare that they have no competing interests.

Funding: This work was supported in part by CRC Multidisciplinary Support (MDS) grant from the Florida State University to QXAS and YR, and an Endowed Chair Professorship in Cancer Research from anonymous donors to QXAS.

Authors' contributions: KAV participated in the study design, performed all the experiments, performed data analysis, and prepared the manuscript. DBB contributed to the study design, analyzed data, and revised the manuscript. YR participated in the study design and helped revise the manuscript. QXAS 
conceived of the study, directed the research, analyzed the data, and helped write and revise the manuscript. All authors read and approved the final manuscript.

Acknowledgements: The authors acknowledge the Tulane University Health Sciences Center (New Orleans, LA) for providing the human mesenchymal stem cells used in this study. The authors wish to thank Dr. Brian Washburn and Kristina Poduch of the Florida State University Molecular Cloning Facility for qRT-PCR assistance. Lastly, the authors wish to thank Youry Pierre-Louis and Samantha Chuck for technical assistance.

\section{References}

1. World Health Organization. Obesity and overweight - Fact Sheet (updated Feb. 2018). Available at: https://www.who.int/en/news-room/fact-sheets/detail/obesity-and-overweight. (Accessed: 15th April 2019)

2. Hales, C. M., Carroll, M. D., Fryar, C. D. \& Ogden, C. L. Prevalence of Obesity Among Adults and Youth: United States, 2015-2016 Key findings Data from the National Health and Nutrition Examination Survey. (2015).

3. Bhaskaran, K. et al. Body-mass index and risk of 22 specifi c cancers: a population-based cohort study of 5·24 million UK adults. Lancet 384, 755-765 (2014).

4. Blair, C. K. et al. Obesity and survival among a cohort of breast cancer patients is partially mediated by tumor characteristics. npj Breast Cancer 5, 1-7 (2019).

5. Calle, E. E., Rodriguez, C., Walker-Thurmond, K. \& Thun, M. J. Overweight, Obesity, and Mortality from Cancer in a Prospectively Studied Cohort of U.S. Adults. N. Engl. J. Med. 348, 1625-1638 (2003).

6. Cao, Y. \& Ma, J. Body mass index, prostate cancer-specific mortality, and biochemical recurrence: A systematic review and meta-analysis. Cancer Prevention Research 4, 486-501 (2011).

7. Dirat, B. et al. Cancer-Associated Adipocytes Exhibit an Activated Phenotype and Contribute to Breast Cancer Invasion. Cancer Res. 71, 2455-2465 (2011).

8. Duong, M. N. et al. The fat and the bad: Mature adipocytes, key actors in tumor progression and resistance. Oncotarget 8, 57622-57641 (2017).

9. Carter, J. C. \& Church, F. C. Mature breast adipocytes promote breast cancer cell motility. Exp. Mol. Pathol. 92, 312-317 (2012).

10. $\mathrm{Xu}, \mathrm{H}$. et al. Chronic inflammation in fat plays a crucial role in the development of obesity-related insulin resistance. J. Clin. Invest. 112, 1821-30 (2003).

11. Weisberg, S. P. et al. Obesity is associated with macrophage accumulation in adipose tissue. J. Clin. Invest. 112, 1796-808 (2003).

12. Suganami, T. \& Ogawa, Y. Adipose tissue macrophages: their role in adipose tissue remodeling. J. Leukoc. Biol. 88, 33-39 (2010). 
13. Qiu, S. Q. et al. Tumor-associated macrophages in breast cancer: Innocent bystander or important player? Cancer Treatment Reviews 70, 178-189 (2018).

14. Mantovani, A., Marchesi, F., Malesci, A., Laghi, L. \& Allavena, P. Tumour-associated macrophages as treatment targets in oncology. Nature Reviews Clinical Oncology 14, 399-416 (2017).

15. Sekiya, I., Larson, B. L., Vuoristo, J. T., Cui, J.-G. \& Prockop, D. J. Adipogenic Differentiation of Human Adult Stem Cells From Bone Marrow Stroma (MSCs). J. Bone Miner. Res. 19, 256-264 (2003).

16. Bosco, D. B., Kenworthy, R., Zorio, D. A. R. \& Sang, Q.-X. A. Human Mesenchymal Stem Cells Are Resistant to Paclitaxel by Adopting a Non-Proliferative Fibroblastic State. PLoS One 10, e0128511 (2015).

17. Sintiprungrat, K., Singhto, N., Sinchaikul, S., Chen, S. T. \& Thongboonkerd, V. Alterations in cellular proteome and secretome upon differentiation from monocyte to macrophage by treatment with phorbol myristate acetate: Insights into biological processes. J. Proteomics 73, 602-618 (2010).

18. Song, M. G. et al. NRF2 signaling negatively regulates phorbol-12-myristate-13-acetate (PMA)induced differentiation of human monocytic U937 cells into pro-inflammatory macrophages. PLoS One 10, e0134235 (2015).

19. Livak, K. J. \& Schmittgen, T. D. Analysis of Relative Gene Expression Data Using Real-Time Quantitative PCR and the 2- $\triangle \Delta C T$ Method. Methods 25, 402-408 (2001).

20. Andarawewa, K. L. et al. Stromelysin-3 Is a Potent Negative Regulator of Adipogenesis Participating to Cancer Cell-Adipocyte Interaction/Crosstalk at the Tumor Invasive Front. Cancer Res. 65, 1086210871 (2005).

21. Lijnen, H. R., Van, H. B., Frederix, L., Rio, M. C. \& Collen, D. Adipocyte hypertrophy in stromelysin-3 deficient mice with nutritionally induced obesity. Thromb. Haemost. 87, 530-5 (2002).

22. Maquoi, E., Demeulemeester, D., Vörös, G., Collen, D. \& Lijnen, H. R. Enhanced nutritionally induced adipose tissue development in mice with stromelysin-1 gene inactivation. Thromb. Haemost. 89, 696-704 (2003).

23. Wang, Y. Y. et al. Adipose tissue and breast epithelial cells: A dangerous dynamic duo in breast cancer. Cancer Letters (2012). doi:10.1016/j.canlet.2012.05.019

24. Solinas, G., Germano, G., Mantovani, A. \& Allavena, P. Tumor-associated macrophages (TAM) as major players of the cancer-related inflammation. J. Leukoc. Biol. 86, 1065-1073 (2009).

25. Booth, A., Magnuson, A., Fouts, J. \& Foster, M. Adipose tissue, obesity and adipokines: role in cancer promotion. Horm. Mol. Biol. Clin. Investig. 21, 57-74 (2015).

26. Hauner, H., Petruschke, T., Russ, M., Röhrig, K. \& Eckel, J. Effects of tumour necrosis factor alpha (TNFa) on glucose transport and lipid metabolism of newly-differentiated human fat cells in cell culture. Diabetologia 38, 764-771 (1995).

27. Petruschke, T. \& Hauner, H. Tumor necrosis factor-alpha prevents the differentiation of human adipocyte precursor cells and causes delipidation of newly developed fat cells. J. Clin. Endocrinol. Metab. 76, 742-747 (1993). 
28. Ahmad, A. et al. Stromelysin 3: An independent prognostic factor for relapse-free survival in nodepositive breast cancer and demonstration of novel breast carcinoma cell expression. Am. J. Pathol. 152, 721-8 (1998).

29. Permana, P. A., Menge, C. \& Reaven, P. D. Macrophage-secreted factors induce adipocyte inflammation and insulin resistance. Biochem. Biophys. Res. Commun. 341, 507-514 (2006).

30. Subbaramaiah, K. et al. Obesity Is Associated with Inflammation and Elevated Aromatase Expression in the Mouse Mammary Gland. Cancer Prev. Res. 4, 329-346 (2011).

31. Subbaramaiah, K. et al. Obesity Is Associated with Inflammation and Elevated Aromatase Expression in the Mouse Mammary Gland. Cancer Prev. Res. 4, 329-346 (2011).

\section{Figures}

A

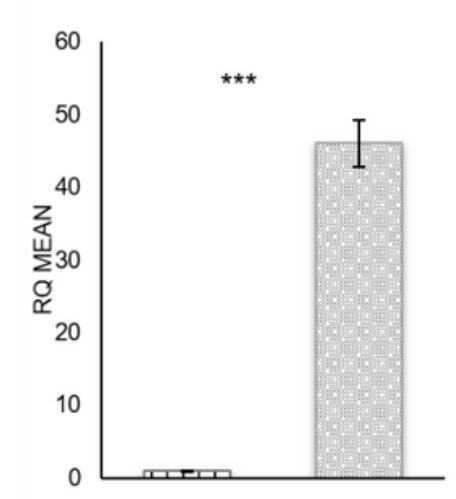

B

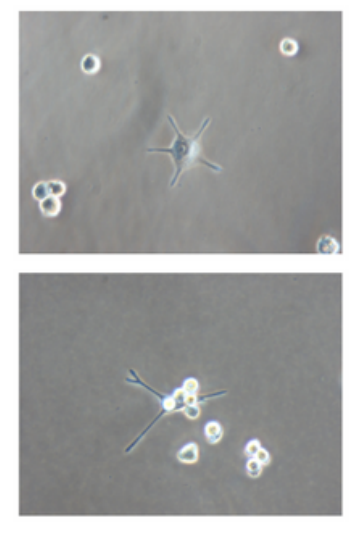

C TNF- $a$

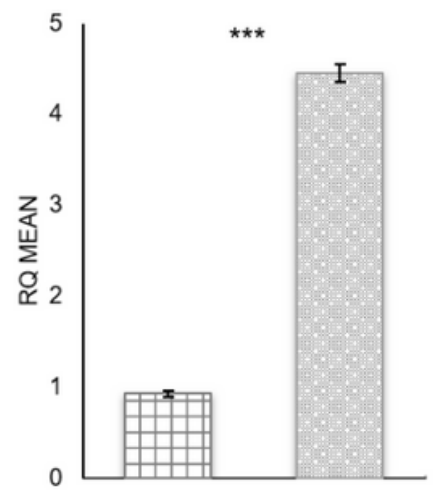

IL-6

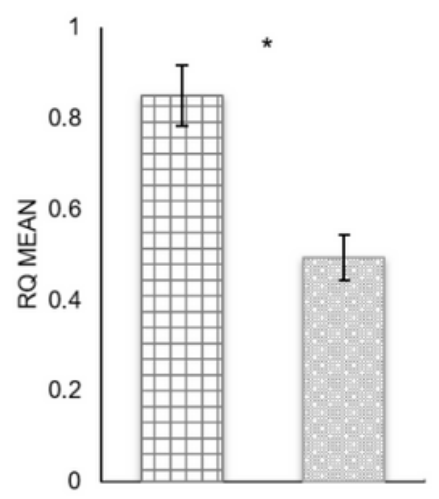

IL-1 $\beta$

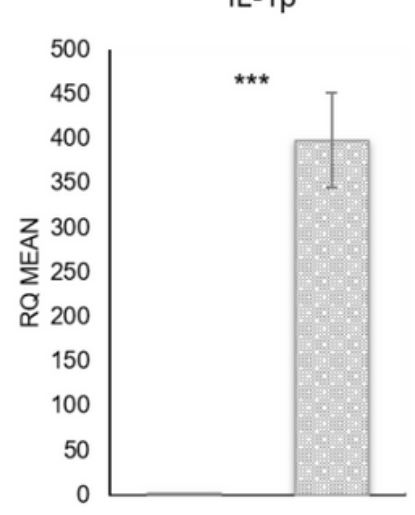

可 U937 Undifferentiated

Macrophage

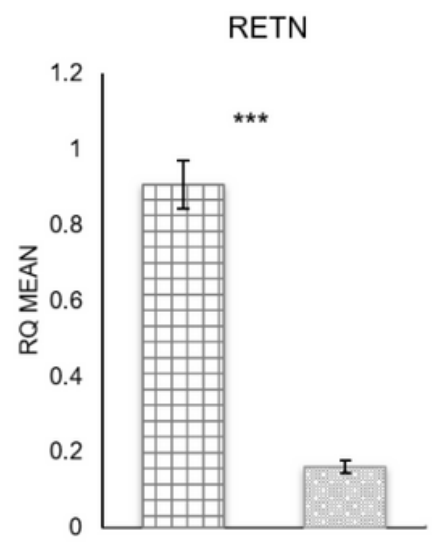

\section{Figure 1}

U937 cell gene expression level measured by qRT-PCR. (A) Expression levels of CD11b in undifferentiated and macrophage differentiated U937 cells. CD11b was significantly upregulated after differentiation. (B) Differentiated cells show morphological changes, from suspension cells to attached, macrophage-like cells. Magnification 200x. (C) TNF- $\alpha$ and IL-1 $\beta$ expression increased after differentiation, while IL- 6 and RETN expression decreased. $R Q$ is relative quantification. * $P \leq 0.05$, ** $P \leq 0.01$, $* \star * P \leq 0.001$. Data presented as mean \pm SEM. 
A AdipoQ

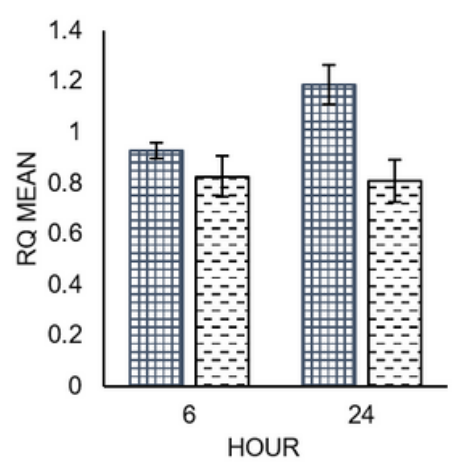

IL-1 $\beta$

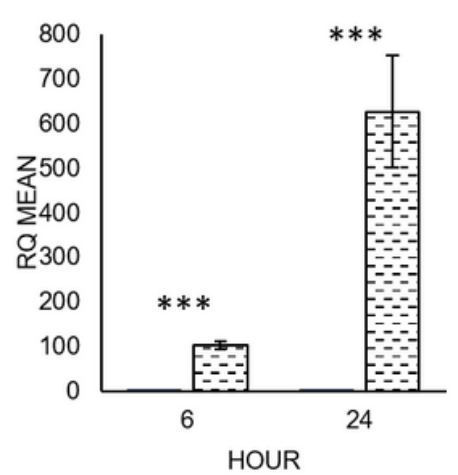

B

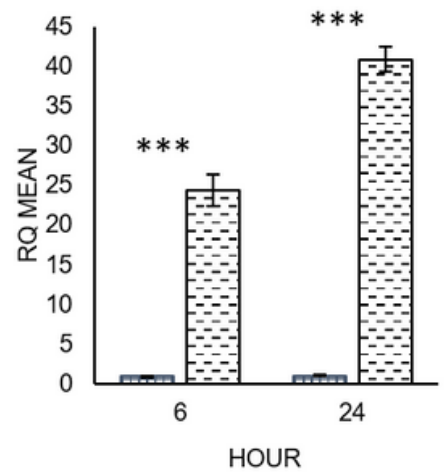

LEP

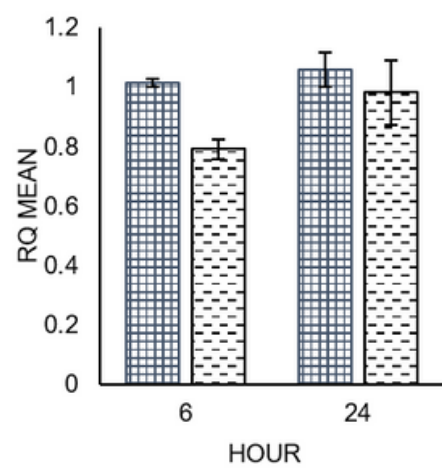

IL-6

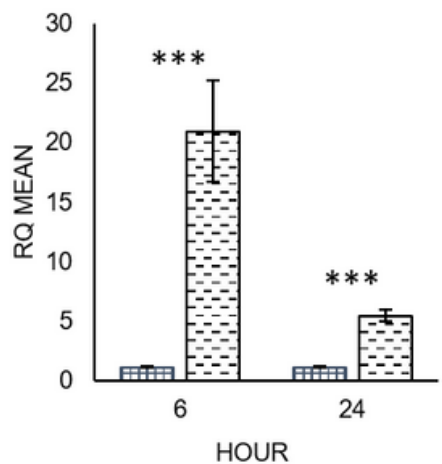

MMP-11

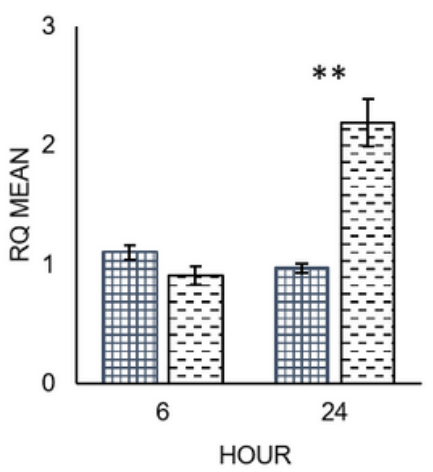

TNF- $\alpha$

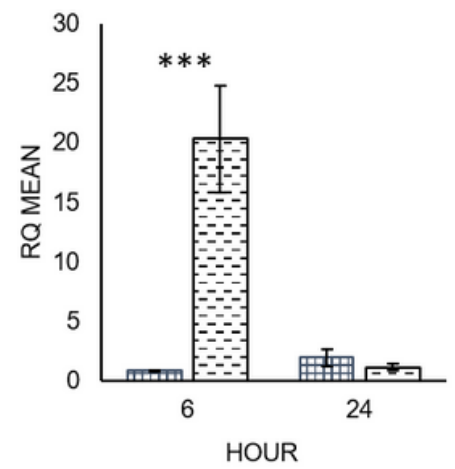

PAl-1
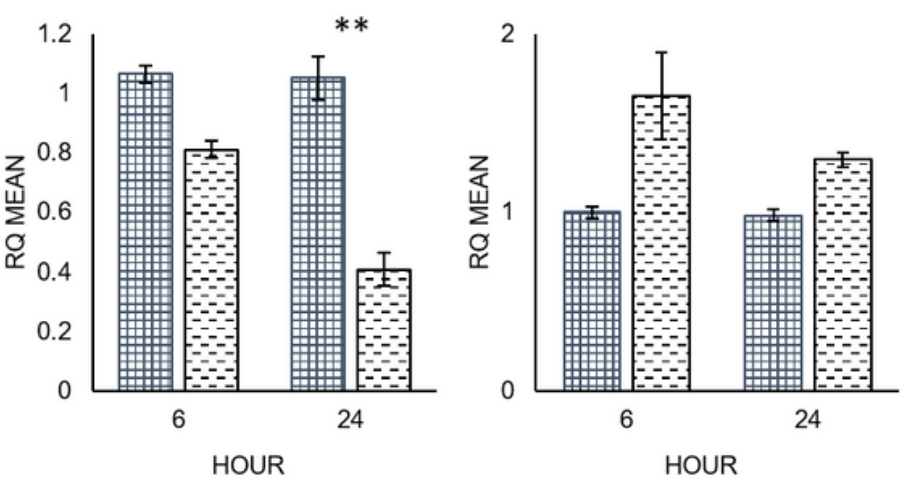

\section{Figure 1}

Cytokine (A) and MMP (B) expression of adipocytes not in co-culture with macrophage $\mathrm{CM}$ or without macrophage $\mathrm{CM}$, at 6 and 24 hours. $R Q$ is relative quantification. ${ }^{*} P \leq 0.05$, $* \star P \leq 0.01, * \star * P \leq 0.001$. Data presented as mean \pm SEM. 

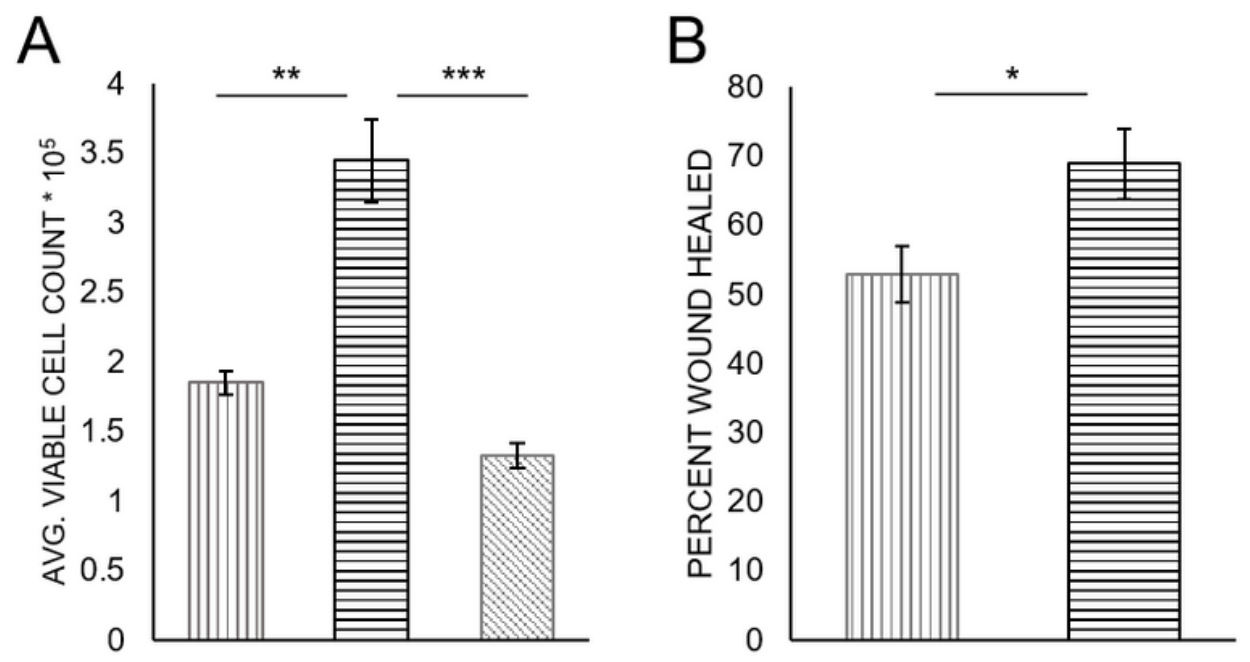
III Co-culture in nonCM
車 Co-culture in CM
Breast cancer cells in $\mathrm{CM}$

\section{Figure 1}

MCF-7 proliferation (A) and wound healing assay (B). Cell counts were used to determine proliferation after 3 days in co-culture with adipocytes, with macrophage $\mathrm{CM}$ or without macrophage $\mathrm{CM}$, or alone in $\mathrm{CM}$. After 3 days in co-culture with adipocytes, with macrophage $\mathrm{CM}$ or without macrophage $\mathrm{CM}$, a scratch was made on the MCF-7 confluent cell layer and migration was measured after 24 hours. * $\mathrm{P} \leq$ $0.05, * \star P \leq 0.01, * \star * P \leq 0.001$. Data presented as mean \pm SEM. 


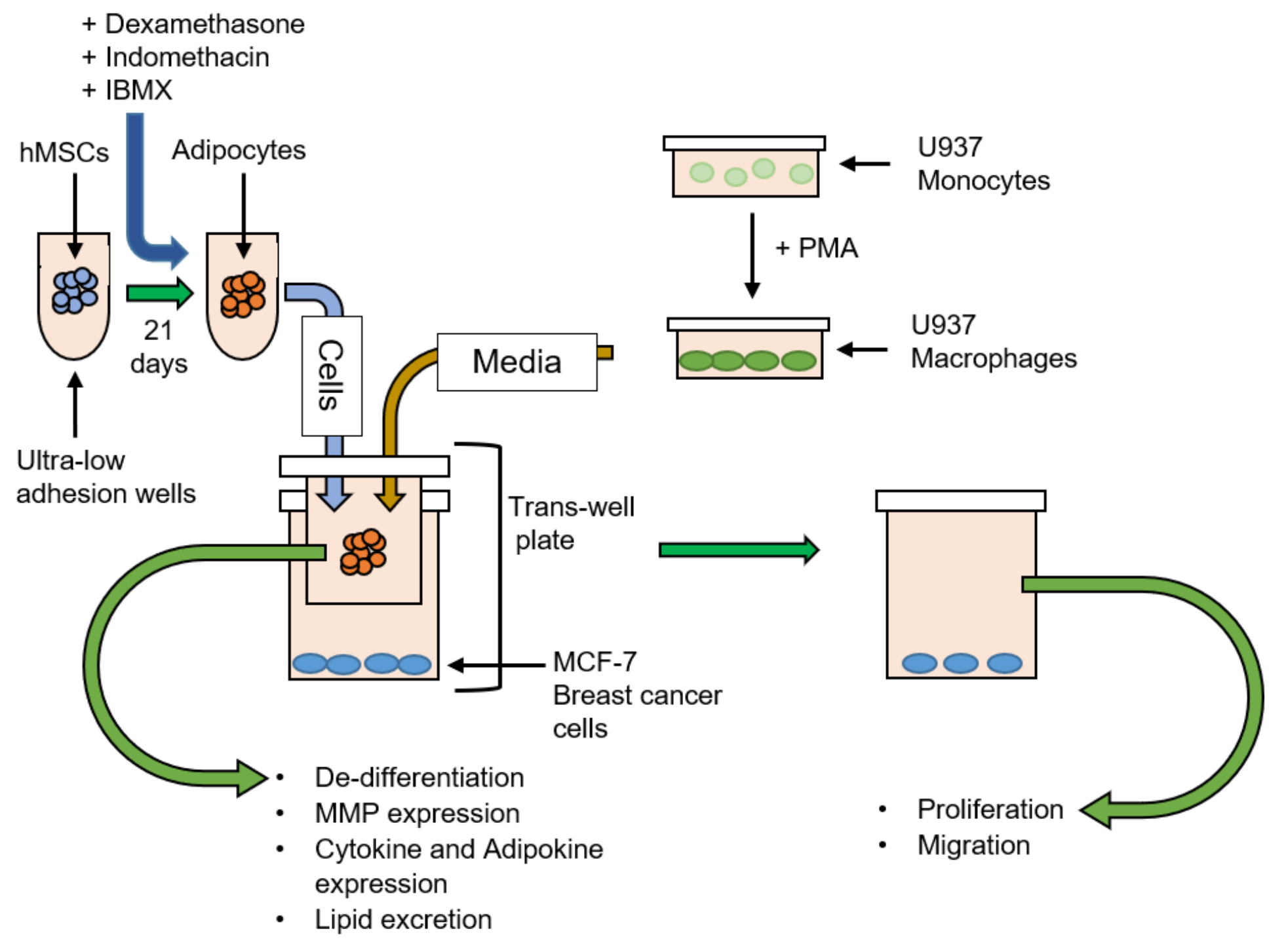

Figure 1

Human cell co-culture scheme. hMSCs were used to make human adipocytes, and placed in co-culture with MCF-7 breast cancer cells. Monocytes were used to make macrophages and conditioned media was used in co-culture. Breast cancer cells were tested for proliferation and migration, while adipocytes were tested for lipid loss and cytokine and MMP expression. 



B

MMP-3

MMP-11
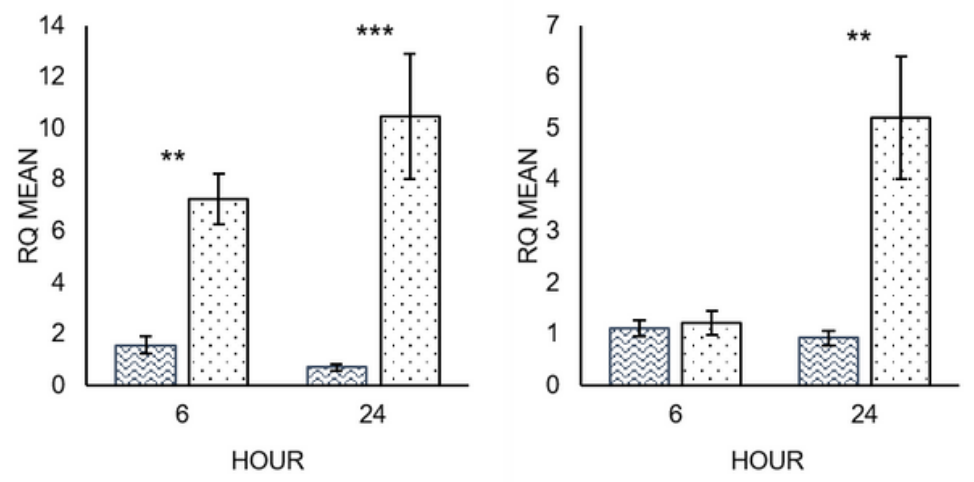

Figure 1

Cytokine (A) and MMP (B) mRNA expression of adipocytes in co-culture with breast cancer cells with macrophage $\mathrm{CM}$ or without macrophage $\mathrm{CM}$, at 6 and 24 hours. $\mathrm{RQ}$ is relative quantification. ${ }^{*} \mathrm{P} \leq 0.05$, $\star \star P \leq 0.01,{ }^{\star * \star} P \leq 0.001$. Data presented as mean \pm SEM.

\section{Supplementary Files}

This is a list of supplementary files associated with this preprint. Click to download.

- Supplementarylnformation.docx 